\title{
Chyle Leak in Head and Neck Cancer Surgeries - A review
}

\section{Sailesh Kumar $\mathbf{R}^{1 *}$, Raja Sethupathy Cheeman ${ }^{1}$, Raghu $\mathbf{K}^{2}$, Bharathraj $\mathbf{K}^{1}$, Rohan Singh chadha ${ }^{3}$}

${ }^{1}$ Assistant Professor, Indira Gandhi Institute of Dental Sciences, Department of Oral and Maxillofacial Surgery, Sri Balaji Vidyapeeth (Deemed to be university)

Puducherry, India

${ }^{2}$ Associate Professor, Indira Gandhi Institute of Dental Sciences, Department of Oral and Maxillofacial Surgery, Sri Balaji Vidyapeeth (Deemed to be university)

Puducherry, India

${ }^{3}$ Post graduate trainee, Indira Gandhi Institute of Dental Sciences, Department of Oral and Maxillofacial Surgery, Sri Balaji Vidyapeeth (Deemed to be university) Puducherry, India

*Corresponding Author: Sailesh Kumar R, Assistant Professor, Indira Gandhi Institute of Dental Sciences, Department of Oral and Maxillofacial Surgery, Sri Balaji Vidyapeeth ( Deemed to be university) Puducherry, India.
Received: January 10, 2022

Published: February 10, 2022

(C) All rights are reserved by Sailesh Kumar

R., et al.

\begin{abstract}
Background: This article projects provides insights to the various managements and treatment strategies for chylous leak during the head and neck cancer surgeries.

Methods: Here the retrospective review of articles was performed, the articles reviewed are involving only head and neck cancer surgeries only and analysis of treatment strategies were noted and author's comments about the treatment strategies is given after collective analysis of the review articles

Conclusion: The evolution of treatment strategies through years has brought on to the ideal management of the of chyle leak based on various authors guidelines.
\end{abstract}

Keywords: Chyle; Chylothorax; Head and Neck Cancer Surgery; Neck Dissection; Chyle Fistula

\section{Abbreviations:}

MCT: Medium Chain Triglycerides; VATS: Video Assisted Thoracic Surgery; TPN: Total Parenteral Nutrition

\section{Introduction}

Head and Neck Cancer surgeries result in a rare complication known as chyle leak or chylothorax, it is a life-threatening condition and requires immediate attention and intervention [1,2].

The normal chyle flow is 1500 - $2500 \mathrm{~mL}$ per day, fasting chyle flow can be low as $10-15 \mathrm{~mL}$ per hour. Production of chyle may vary with meal and activity of bowel function too [4]. Chyle production increases with any intake by enteral route including water thereby increasing thoracic duct flow.

Concentrations greater than $1.2 \mathrm{mmol} / \mathrm{L}$ of chylomicrons in the pleural fluid are considered to be indicative of chylothorax [4]. The accumulation of chyle from the thoracic duct into the pleural space is called as Chylothorax [1]. During any head and neck surgeries an obstruction or any damage leading to laceration of the thoracic duct will result in formation of chylothorax. Anatomical variations in the course of lymphatic system leads to higher chance of surgical trauma [3]. Chylothorax if left untreated by very alarming and can lead to reduced sodium content and decreases the fluid and electrolyte contents in the body, also can cause immuno-compromised state [5].

Chyle leak management with review

Management of chyle leak is predominantly done by two methods either by conservative methods or by surgical management. By conservative therapy the fat intake is reduced or stopped by starting with proper enteral or by parenteral feeding diet, thereby decreases the chyle flow [6,7]. Frequent postural changes has to be avoided to decrease the outflow of chyle, so bed rest is advised 
$[9,10]$. Diet with Medium-chain triglycerides (MCTs) is recommended $[9,10,11,28,29]$. MCTs do not get absorbed in lymphatics and thereby do not form chyle outflow and some of the authors have suggested this diet with MCT can be the preferred treatment of choice in chyle leak $[10,28,30]$.

If MCT does not give results, then Total Parenteral Nutrition (TPN) is suggested by authors [9]. TPN produces phospholipids directly to the central venous circulation, it also has risks of catheter infection, also sometimes metabolic and electrolyte imbalance/ disturbances can occur [9,32,33] and it is costlier in comparison to enteral feeding. Somatostatin along with its synthetic analogue octreotide produces decreased chyle production [34-37]. It produces results within two weeks of usage in low to moderate cases of chyle leak $[35,37]$. Orlistat which is an pancreatic lipase inhibitor is found to be successful in treatment of low-volume chyle leakage [38].

Chyle leak can be surgically managed either at local or at a distant site. Local procedures were done to block the chyle flow at original operational site or the distant procedure involves occluding the flow of chyle in distal thoracic duct. Fat when ingested preoperatively helps in identification of the leak [16]. The inflammation caused due to the chyle leak can hinder identification of major vessels and nerves in the region, therefore during surgical management caution should be practised $[9,10,14]$.

Many authors have preferred for usage of the ligation clips and non-absorbable suture material for surgical management of chyle leak $[9,10,26]$. Coverage of the area is recommended either with a local flap using pectoralis major or the clavicular head of sternocleidomastoid muscle if it was not removed, this should be further supplemented with application of sclerosing or bonding substances, placement of vicryl mesh was also described $[9,13,24]$.

At the chyle leak site, surgical reoperation is difficult as the anatomy will vary due to scarring and there is more risk in damage of vital structures. Earlier authors used to believe that ligation of the thoracic duct to be fatal, Stuart., et al. in early 1900s hypothesized that the collateral lymph channels can take over the lymph flow [39]. Transthoracic access technique of Lampson is one the surgical methods used for accessing and ligating the thoracic duct [8].

The concept of transabdominal cannulation of the duct was pioneered to provide a non-invasive alternative to the surgical re-op- eration of the thoracic duct [27]. The transabdominal canulation of the cisterna chyli by pedal lymphangiography helps in delineating the lymphatic vessels thereby allowing the surgeon to use embolization coils or histocryl (N-butyl-2-cyanoacrylate) tissue adhesive [27-29]. This method provides a success rate of $60 \%$ in postoperative chylothorax [28].

In cases where the patient had no response to conservative management of cervical chyle fistulas Van Goor., et al. adopted this technique of transabdominal cannulation technique and they found it to be time consuming and requires several attempts [29]. If chyle leak was detected and ligated during head and neck surgery, close monitoring is recommended during post operative period to assess for leakage [10]. Greasy fluid in drain should be doubted for a chyle leak and for further confirmatory diagnosis triglyceride analysis should be done [10].

Various literatures sub classifies the chyle fistulas into low and high outflow. High output - ranging from $>500 \mathrm{ml} /$ day to $>1000$ $\mathrm{ml} /$ day [11]. The high outflow chyle does not respond well to conservative management than the low outflow cases as documented by many authors $[11,16]$. According to the recommendations given by some authors bed rest and immediately start with Naso-Gastric feeding with MCTs in cases of chyle fistula, following which the vitals, fluid - electrolyte balance should be monitored, Liver function test to be performed periodically done to monitor any pathological response [10,16,29].

If there is a vascular compromise to a free flap in such scenarios early thoracoscopic ligation is indicated and any fresh reconstruction plans should be delayed until the chyle flow is stemmed [32]. If the chyle flow is persistent for more than five days then the patient should be taken up for surgical management as suggested by some authors, thereby the method of thoracoscopic ligation is advised than exploration of the suspicious leak site or continuing the conservative management in such cases $[11,30,40]$.

The surgical procedure of thoracotomy has recently been evolved to Video assisted thoracic surgery (VATS) or Robotic approach, recently VATS has been the treatment of choice in surgery of thoracic duct $[5,33,34]$.

In scenarios where the leak is not been able to be identified then the mass ligation of the thoracic duct is indicated above the esophageal hinge between the aorta, pericardium and vertebral bodies, 
thereby ensuring the ligation at its entry in chest and also all the accessory ducts are all sealed. This mass ligation could be performed by thoracoscopy [35]. The landmark suggested by some authors is to ligate the thoracic duct behind the cisterna chyli by abdominal approach [36].

Radiotherapy is an alternative therapy for the treatment of chylothorax postoperatively, this treatment is proposed by some authors based on successful management of inguinal lymphatic fistulas by radiotherapy.

$[37,38]$. Free fat diet in combination with Radiation therapy could provide an effective alternate therapy for the surgical management, less evidence supports the reliability but it could still be effective [39].

\section{Conclusion}

The Management of chylothorax is challenging but according to the above review of articles it is clear about the various strategic management with the recent technologies like VATS, radiotherapy and dietary management. Meticulous neck dissection during head and neck onco-surgeries should avoid chyle leak, but the chance of the chyle leak produced is unpredictable and the operating surgeon should be well prepared to diagnose and manage the leak effectively during intra operative and post operative period.

\section{Conflict of Interest}

No Conflict of interest.

\section{Bibliography}

1. Morris SA and Taylor SJ. "Peripheral parenteral nutrition in a case of chyle leak following neck dissection". Journal of Human Nutrition and Dietetics 17 (2004): 153-155.

2. Lee., et al. "Management of chylous leakage following neck dissection - cases report". Journal of Dental Sciences 3.1 (2008).

3. Vallieres E., et al. "Early complications Chylothorax". Chest Surgery Clinics of North America 9 (1999): 609-616.

4. Teba L., et al. "Chylothorax review". Critical Care Medicine13 (1985): 49-52.

5. Martucci., et al. "Postoperative chylothorax". Thoracic Surgery Clinics 25 (2015): 523-528.

6. Chalret du Rieu M., et al. "Management of postoperative chylothorax". Journal of Visceral Surgery 148 (2011): e346-352.
7. Riquet M., et al. "Traitment du chylothorax". EMC-Chirurgie 6 (2004): 662-681.

8. Selle JG., et al. "Chylothorax: indications for surgery". Annals of Surgery 177 (1973): 245-249.

9. Henril^tte HW., et al. "Systematic approach to the treatment of chylous leakage after neck dissection”. Head Neck 18.4 (1996): $347 \mathrm{e} 51$.

10. Lucente FE., et al. "Chyle fistula management”. OtolaryngologyHead and Neck Surgery 89.4 (1981): 575e8.

11. Nussenbaum B., et al. "Systematic management of chyle fistula: the southwestern experience and review of the literature". Otolaryngology-Head and Neck Surgery 122.1 (2000): 31e8.

12. Fitz-Hugh GS and Cowgill R. "Chylous fistula”. Archives of Otolaryngology 91.6 (1907): 543e7.

13. Qureshi SS and Chaturvedi P. "A novel technique of management of high output chyle leak after neck dissection". Journal of Surgical Oncology 96.2 (2007): $176 \mathrm{e} 7$.

14. Gregor RT. "Management of chyle fistulization in association with neck dissection". Otolaryngology-Head and Neck Surgery 122.3 (2000): 434e9.

15. Crumley RL and Smith JD. "Postoperative chylous fistula prevention and management”. Laryngoscope 86.6 (1976): 804e13.

16. Jeffrey D., et al. "The management of chyle fistula". Laryngoscope 100.7 (1990): 771e4.

17. Martin IC., et al. "Medium chain triglycerides in the management of chylous fistulae following neck dissection". British Journal of Oral and Maxillofacial Surgery 31.4 (1993): 236e8.

18. Al-Khayat M., et al. "Nutritional support in patients with low volume chylous fistula following radical neck dissection". The Journal of Laryngology and Otology 105.12 (1991): 1052e6.

19. McGee DC and Gould MK. "Preventing complications of central venous catheterization". The New England Journal of Medicine 348.12 (2003): 1123e33.

20. Knochel JP. "Complications of total parenteral nutrition". Kidney International 27.3 (1985): 489e96.

21. Angelico $M$ and Guardia PD. "Review article: hepatobiliary complications associated with total parenteral nutrition". Alimentary Pharmacology and Therapeutics 14 (2000): 54e7. 
22. Rimensberger PC., et al. "Treatment of a persistent postoperative chylothorax with somatostatin". The Annals of Thoracic Surgery 66.1 (1998): 253e4.

23. Valentine CN., et al. "Somatostatin analog treatment of a cervical thoracic duct fistula”. Head Neck 24.8 (2002): 810e3.

24. Nyquist GG., et al. "Octreotide in the medical management of chyle fistula". Otolaryngology-Head and Neck Surgery 128.6 (2003): 910e1.

25. Belloso A., et al. "The community management of chylous fistula using a pancreatic lipase inhibitor (orlistat)". Laryngoscope 116.10 (2006): $1934 \mathrm{e} 5$.

26. Stuart WJ. "Operative injuries of the thoracic duct in the neck". Edinburgh Medical Journal 22 (1907): 301e17.

27. Cope C. "Diagnosis and treatment of postoperative chyle leakage via percutaneous transabdominal catheterization of the cisterna chyli: a preliminary study". Journal of Vascular and Interventional Radiology 9.5 (1998): 727e34.

28. Cope C. "Management of chylothorax via percutaneous embolization". Current Opinion in Pulmonary Medicine 10.4 (2004): $311 \mathrm{e} 4$.

29. van Goor AT., et al. "Introduction of lymphangiography and percutaneous embolization of the thoracic duct in a stepwise approach to the management of chylous fistulas". Head Neck 29.11 (2007): 1017e23.

30. Lapp GC., et al. "Thoracoscopic management of chylous fistulae". American Journal of Otolaryngology 1998;19.4 (1998): $257 \mathrm{e} 62$.

31. Van Natta TL., et al. "Thoracoscopic thoracic duct ligation for persistent cervical chyle leak: utility of immediate pathologic confirmation". JSLS 13.3 (2009): 430e2.

32. Abdel-Galil K., et al. "High output chyle leak after neck surgery: the role of video-assisted thoracoscopic surgery". British Journal of Oral and Maxillofacial Surgery 47.6 (2009): 478e80.

33. Rocco G. Chylothorax. In: "Sabiston DC, Spencer EC, editors. Surgery of the chest. 8th edition. Philadelphia: WB Saunders (2010): 427-430.

34. Thompson KJ., et al. "Treatment of chylothorax by robotic thoracic duct ligation". The Annals of Thoracic Surgery 85 (2008): 334-336.
35. Kent RB $3^{\text {rd }}$ and Pinson TW. "Thoracoscopic ligation of the thoracic duct”. Surgical Endoscopy 7 (1993): 52-53.

36. Mason PF., et al. "Post-thoracotomy chylothorax - a cure in the abdomen?" European Journal of Cardio-Thoracic Surgery 11 (1997): 567-1570.

37. Dietl B., et al. "Radiotherapy of inguinal lymphorrhea after vascular surgery. A retrospective analysis". Strahlentherapie und Onkologie 181 (2005): 396-400.

38. Gerstein J., et al. "Complete remission of a lymphoma-associated chylothorax by radiotherapy of the celiac trunk and thoracic duct". Strahlentherapie und Onkologie 2008;184 (2008): 484-487.

39. Sziklavari Z., et al. "Radiotherapy in the treatment of postoperative chylothorax". Journal of Cardiothoracic Surgery 8 (2013): 72.

40. Andrei Ilczyszyn., et al. "Management of chyle leak post neck dissection: A case report and literature review". Journal of Plastic, Reconstructive and Aesthetic Surgery 64 (2011): e223ee230.

\section{Assets from publication with us}

- Prompt Acknowledgement after receiving the article

- Thorough Double blinded peer review

- Rapid Publication

- Issue of Publication Certificate

- High visibility of your Published work

Website: www.actascientific.com/

Submit Article: www.actascientific.com/submission.php

Email us: editor@actascientific.com

Contact us: +919182824667 